\title{
Observations on the phylogeny of Opisthadena Linton, 1910 and related genera (Hemiuridae: Opisthadeninae) from Australian and French Polynesian waters
}

\author{
Rodney A. Bray ${ }^{1}$ and Thomas H. Cribb ${ }^{2}$ \\ ${ }^{1}$ Department of Zoology, The Natural History Museum, Cromwell Road, London SW7 5BD, UK; \\ ${ }^{2}$ Centre for Marine Studies and Department of Microbiology and Parasitology, The University of Queensland, Brisbane, \\ Queensland 4072, Australia
}

Key words: Hemiuridae, Opisthadena, Mitrostoma, Neopisthadena, Aplodactylus, Kyphosus, Great Barrier Reef, Southwestern Australia, French Polynesia

\begin{abstract}
Mitrostoma nototheniae Manter, 1954 is redescribed from Aplodactylus arctidens Richardson, off northern Tasmania. Opisthadena dimidia Linton, 1910 is reported from Kyphosus bigibbus Lacepède, Ningaloo, Western Australia, K. cinerascens (Forsskål), off Heron Island and Ningaloo, Western Australia, Kyphosus cornelii (Whitley), off Kalbarri, Western Australia, K. sydneyanus Günther, off Fremantle, Western Australia, K. sydneyanus ?, Ningaloo, Western Australia and K. vaigiensis (Quoy et Gaimard), off Heron and Lizard Islands, Queensland and Moorea, French Polynesia: measurements and an illustration are given. Neopisthadena habei Machida, 1980 is reported from K. sydneyanus off Fremantle, Western Australia: measurements and an illustration are given. Data derived from these specimens are used to recode a data-matrix developed by León-Règagnon et al. (1996), and the resultant tree produced is almost congruent with that of these authors. Our data indicate that this group of parasites is associated mostly with herbivorous hosts and that $O$. dimidia, while geographically widespread, is stenoxenic to the genus Kyphosus.
\end{abstract}

The subfamily Opisthadeninae Yamaguti, 1970 was considered by Gibson and Bray (1979) to include the four genera Opisthadena Linton, 1910, Genolinea Manter, 1925, Mitrostoma Manter, 1954 and Neotheletrum Gibson et Bray, 1979, and to belong in the family Bunocotylidae Dollfus, 1950. Brooks et al. (1985) reverted to the earlier opinion that the taxon should be recognised as Bunocotylinae, a subfamily of the Hemiuridae Looss, 1899. The results of LeónRègagnon et al. (1998) supported the clade formed by the constituent genera of the Opisthadeninae (now also including Neopisthadena Machida, 1980) and Gibson (2002) retained the taxon Opisthadeninae, but recognised it as a subfamily of the Hemiuridae. LeónRègagnon et al. (1996) recently presented a cladistic analysis of Opisthadena, using as outgroups the other genera of the Opisthadeninae. Our collecting in the waters around Australia and in French Polynesia has produced members of three of these genera from new hosts and localities. We are reporting here these findings and using this new material to refine the evolutionary hypothesis proposed by León-Règagnon et al. (1996). In the case of Mitrostoma nototheniae Manter, 1954, reported here for only the second time, we present a full description. The other species are better known and, therefore, we are giving only illustrations and measurements.

\section{MATERIALS AND METHODS}

Digeneans collected from freshly killed fishes were fixed by being pipetted into nearly boiling saline and immediately preserved in $2 \%$ formaldehyde or $70 \%$ ethanol, or fixed in Berland's fluid and preserved in $80 \%$ ethanol. Whole-mounts were stained with Mayer's haematoxylin or Mayer's paracarmine, cleared in methyl salicylate or beechwood creosote and mounted in Canada balsam. Measurements were made through a drawing tube on an Olympus BH-2 microscope, using a Digicad Plus digitising tablet and Carl Zeiss KS100 software adapted by Imaging Associates, and are quoted in micrometres. The following abbreviations are used: BMNH, the British Museum (Natural History) collection at The Natural History Museum, London, UK; QM, Queensland Museum collection, Brisbane, Australia. Fish synonymy is based on Froese and Pauly ( 2001).

The cladistic analyses were performed in PAUP* (Swofford 1998), using the exhaustive search option; 21 unordered characters of eight species were analysed, 1000 heuristic bootstrap replicates were performed.

\section{RESULTS}

Family: H e mi u rid a e Looss, 1899

Subfamily: O p i s th a d e n in a e Yamaguti, 1970

Mitrostoma nototheniae Manter, 1954

Fig. 1

Description. Based on two whole-mounts. Measurements in Table 1. Body narrows anteriorly, truncate 
posteriorly (Fig. 1). Tegument unarmed, with fold around ventral sucker and transverse fold in anterior hindbody. Pre-somatic pit in posterior forebody, opens on surface as transverse slit. Pre-oral lobe forms hoodlike fold, bears row of about 5 small papillae. Oral sucker subglobular, subterminal. Ventral sucker transversely oval, in anterior third of body; aperture surrounded by distinct sphincter. Pharynx subglobular. Oesophagus short or practically absent. Intestinal bifurcation in mid-forebody. Caeca blind, extend close to posterior extremity.

Testes 2, oval, entire, tandem, separated by uterus, in anterior half of hindbody, pre-ovarian. Seminal vesicle tubular, coiled, reaches into posterior half of distance between anterior testis and ventral sucker, fairly thinwalled. Pars prostatica long, almost rectilinear, reaching from distinctly within hindbody to almost sinus-sac in forebody, ensheathed in gland-cells. Ejaculatory duct not detectable. Sinus-sac small, thin-walled, oval, in mid-forebody. Hermaphroditic duct convoluted in sinussac. Sinus-organ not detected. Genital atrium absent. Genital pore median, bifurcal.

Ovary oval, post-testicular, separated from posterior testis by uterus. Seminal receptacle blind, antero-dorsal to ovary. Mehlis' gland dorsal to ovary. Uterus passes posteriorly to vitellarium, then forward sinistrally to ovary, between ovary and posterior testis, sinistrally to posterior testis, between testes, dextral to anterior testis, then sinistrally to seminal vesicle and pars prostatica, narrows dorsally to ventral sucker and passes towards sinus-sac; short distinct metraterm joins male duct in small chamber at base of sinus-sac. Eggs numerous, small, tanned, operculate. Vitellarium with two irregularly globular masses, immediately post-ovarian, oblique.

Excretory pore dorsally subterminal. Vesicle passes sinistrally to ovary and posterior testis, between testes, dextral to anterior testis, bifurcates immediately pretesticular; arms unite dorsally to pharynx.

T y p e-hos t a n d 1 o c a 1 i t y : 'Notothenia macrocephala', Wellington, New Zealand.

$\mathrm{New}$ records:

ex Aplodactylus arctidens Richardson (Perciformes: Aplodactylidae). Stomach. Stanley, Tasmania $\left(40^{\circ} 46^{\prime}\right.$ S, $145^{\circ} 20^{\prime} \mathrm{E}$, Jan. 2000). QM G217862, BMNH 2002.4.18.20. Records :

1. Manter (1954); 2. Present study.

Descriptions: 1,2 .

D e f i n i t i v e ho s t : Perciformes: Aplodactylidae: Aplodactylus arctidens ['Notothenia macrocephala', probably an error, see below] $(1,2)$.

D i s t r i b u ti o n : FAO Area 57 off Tasmania (2); 81 Off New Zealand (1).

Remarks. Manter (1954) described this species from the caeca and intestine of 'Maori chief Notothenia macrocephala Günther', off Wellington, New Zealand. According to Froese and Pauly (2001) N. macro- cephalus is a junior synonym of Paranotothenia magellanica (Forster), and 'Maori chief' is an American common name for this species. Ours is only the second record of Mitrostoma nototheniae and the first from Aplodactylus arctidens and Tasmania. Evidence is accumulating that Manter's host identification may be erroneous. From Tasmanian A. arctidens we have also recovered specimens of the enenterids Proenenterum isocotylum Manter, 1954 and P. ericotylum Manter, 1954, and the fellodistomid Choanomyzus tasmaniae Manter et Crowcroft, 1950. The former two species were originally recorded from 'Notothenia macrocephala' from New Zealand and Manter (1954) reported C. tasmaniae from 'Notothenia macrocephala' from New Zealand. This latter species was originally described from Tasmania in A. arctidens (as Dactylosargus arctidens) (Manter and Crowcroft 1950). We suggest, therefore, that the evidence is strong that somehow Manter mistook the identity of the hostspecies in these cases and that none of these digeneans are to be found in $P$. magellanica.

We can detect no significant differences between our specimens and those described by Manter (1954). He figured a 'nipple-shaped protuberance' at the posterior extremity of the 'type' specimen, but considered that this was an 'abnormality'. Using this new material we have recoded this species in the matrix of LeónRègagnon et al. (1996). Our coding differs in: Character (Ch.) 6, where oral papillae have been seen; Ch. 7, where we find the testes to be tandem; Ch. 8, where we find both character states; Ch. 9, where we find the vitellarium and ovary in the posterior third of the body; Ch. 12, where we find the pars prostatica to reach into the forebody; and $\mathrm{Ch}$. 19, where we find that the excretory vesicle bifurcates immediately anterior to the testes. The eggs in $M$. nototheniae are 23-27 long (according to Manter 1954) and 22-31 long according to our measurements, so fit completely into neither states of character 2 . They do, however, overlap into state 0 , but not state 1 , so the former coding is retained.

Opisthadena dimidia Linton, 1910

Fig. 2

Syns O. cortesi Bravo-Hollis, 1966; O. kyphosi Yamaguti, 1970; Opisthadena sp. of Cribb et al. (2001)

T y p e-hos t a n d 1 o c a 1 i t y : Kyphosus sectatrix, off Dry Tortugas, Florida.

$\mathrm{New}$ records:

ex Kyphosus bigibbus Lacepède, Kyphosidae. Stomach. Ningaloo, Western Australia $\left(22^{\circ} 42^{\prime} \mathrm{S}, 113^{\circ} 40^{\prime} \mathrm{E}\right.$, April 2000). QM G217863-217865, BMNH 2002.4.18.1-2.

ex Kyphosus cinerascens (Forsskål), Kyphosidae. Stomach.

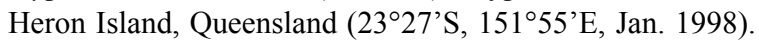
QM G217866-217867, BMNH 2002.4.18.7.

ex Kyphosus cinerascens (Forsskål), Kyphosidae. Stomach.

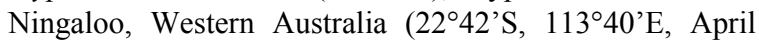
2000). QM G217868-217872, BMNH 2002.4.18.3-6. 

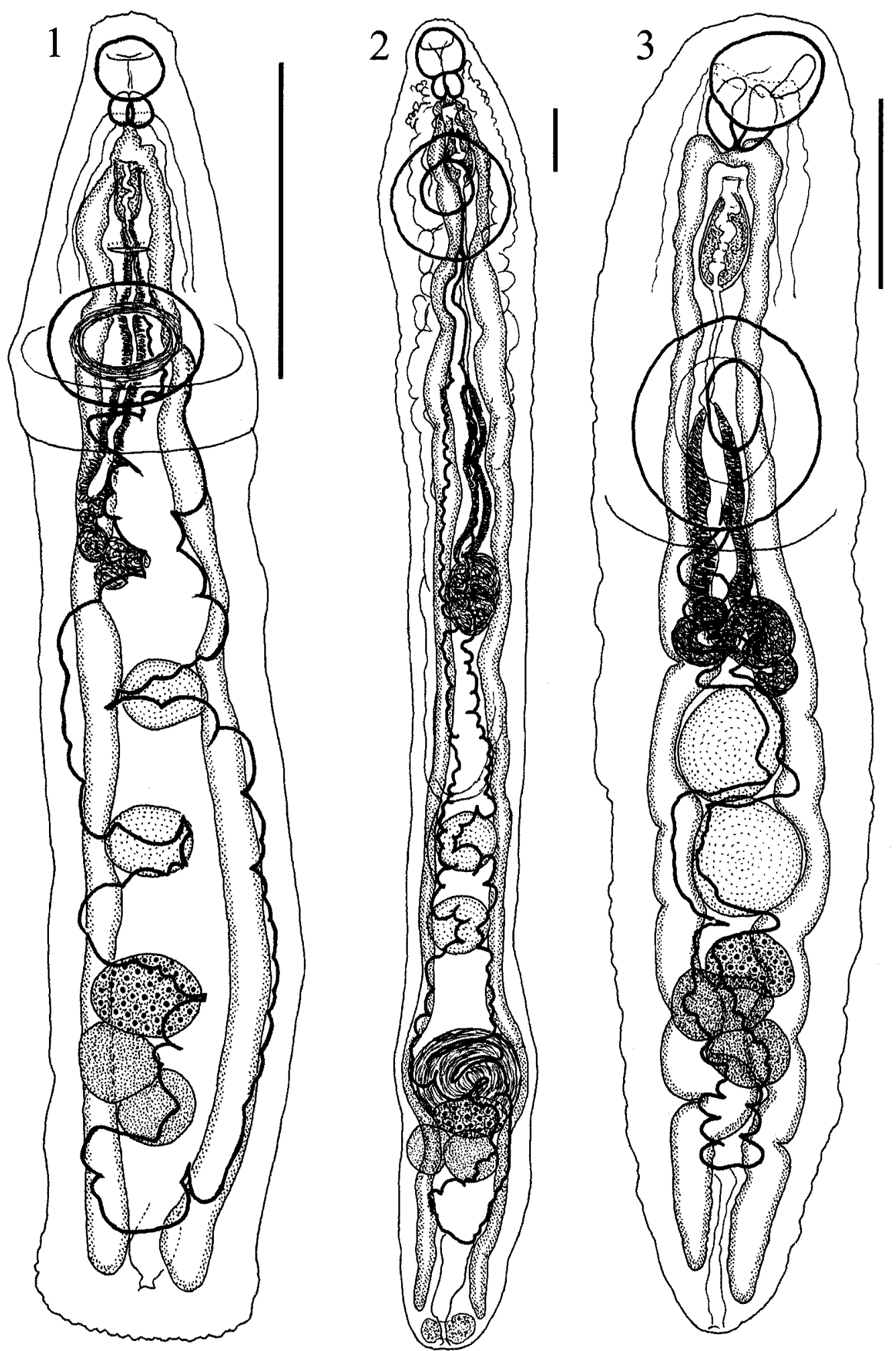

Fig. 1. Mitrostoma nototheniae Manter, 1954. Ventral view, suckers and uterus in bold outline, uterus passes ventrally to gonads but is treated as transparent, posterior and anterior extremities of excretory system only shown. Fig. 2. Opisthadena dimidia Linton, 1910 ex Kyphosus vaigiensis, Moorea, French Polynesia. Ventral view, suckers and uterus in bold outline, uterus passes ventrally to gonads but is treated as transparent, posterior and anterior extremities and bifurcation of excretory system only shown. Fig. 3. Neopisthadena habei Machida, 1980. Ventral view, suckers and uterus in bold outline, uterus passes ventrally to gonads but is treated as transparent, posterior and anterior extremities of excretory system only shown. Scale bars $=500 \mu \mathrm{m}$. 
Table 1. Dimensions of Opisthadena, Mitrostoma and Neopisthadena spp.

\begin{tabular}{|c|c|c|c|}
\hline $\begin{array}{l}\text { Species } \\
\text { Host } \\
\text { Locality } \\
\mathrm{n}\end{array}$ & $\begin{array}{c}\text { Opisthadena dimidia } \\
\text { Kyphosus vaigiensis } \\
\text { Heron Island } \\
8\end{array}$ & $\begin{array}{l}\text { Opisthadena dimidia } \\
\text { Kyphosus vaigiensis } \\
\text { Lizard Island } \\
2\end{array}$ & $\begin{array}{c}\text { Opisthadena dimidia } \\
\text { Kyphosus vaigiensis } \\
\text { Moorea } \\
5\end{array}$ \\
\hline Length & $4,254-8,623(6,229)$ & $1,464-5,492$ & $4,049-8,571(6,212)$ \\
\hline Width & $602-1,182(885)$ & $553-633$ & $515-969(709)$ \\
\hline Forebody & $490-875(623)$ & $424-492$ & $439-774(621)$ \\
\hline Pre-oral lobe & $16-51(33)$ & $28-45$ & $22-85(51)$ \\
\hline Oral sucker & $200-372 \times 164-343(255 \times 233)$ & $189-215 \times 157-197$ & $175-309 \times 167-310(229 \times 228)$ \\
\hline Pharynx & $101-228 \times 106-204(143 \times 154)$ & $104-140 \times 107-133$ & $98-187 \times 114-206(138 \times 151)$ \\
\hline Oesophagus & $47-90(59)$ & $5-12$ & $50-90(66)$ \\
\hline IB to VS & $92-280(161)$ & $133-184$ & $112-252(170)$ \\
\hline Sinus sac & $86-279 \times 95-221(170 \times 155)$ & $120-177 \times 109-138$ & $126-274 \times 110-228(193 \times 156)$ \\
\hline GP to VS & $70-203(126)$ & $98-115$ & $98-206(165)$ \\
\hline Ventral sucker & $474-1,011 \times 477-957(694 \times 682)$ & $451-570 \times 447-561$ & $423-790 \times 411-756(575 \times 568)$ \\
\hline VS to SV & 889-1,528 $(1,238)$ & $795-954$ & $792-1,926(1,336)$ \\
\hline Seminal vesicle & $210-532 \times 163-354(345 \times 243)$ & $201-340 \times 142-203$ & $202-508 \times 154-313(322 \times 220)$ \\
\hline $\mathrm{SV}$ to AT & $511-1,457(781)$ & $807-1,034$ & $548-1,199(915)$ \\
\hline Anterior testis & $191-344 \times 215-393(259 \times 306)$ & $200-238 \times 173-228$ & $173-396 \times 190-373(264 \times 268)$ \\
\hline Distance between testes & $41-339(178)$ & $63-176$ & $58-210(151)$ \\
\hline Posterior testis & $215-369 \times 219-421(279 \times 325)$ & $209-248 \times 184-251$ & $193-379 \times 208-403(267 \times 291)$ \\
\hline $\mathrm{PT}$ to $\mathrm{Ov}$ & $158-697(437)$ & $299-307$ & $292-936(529)$ \\
\hline Ovary & $186-361 \times 253-452(262 \times 341)$ & $215-258 \times 261-290$ & $172-300 \times 221-452(231 \times 315)$ \\
\hline Vitelline masses & $170-343 \times 150-383(252 \times 258)$ & $182-226 \times 160-225$ & $162-331 \times 132-345(237 \times 219)$ \\
\hline Post-vitelline region & $514-1,009(752)$ & $506-687$ & $478-1,092(768)$ \\
\hline Post-uterine region & $331-542(453)$ & $380-447$ & $339-709(457)$ \\
\hline Post-caecal region & $82-228(134)$ & $82-182$ & $49-259(137)$ \\
\hline Eggs & $32-43 \times 12-18(36 \times 14)$ & $31-42 \times 15-22$ & $27-41 \times 11-15(33 \times 13)$ \\
\hline Width \%* & $13-16(14)$ & 12 & $11-13(12)$ \\
\hline Forebody $\% *$ & $8.8-12(10)$ & $9.0-9.5$ & $8.7-13(10)$ \\
\hline Sucker width ratio & $1: 2.7-3.2(2.9)$ & $1: 2.8$ & $1: 2.4-2.6(2.5)$ \\
\hline OS to Pharynx ratio & $1: 1.4-1.7(1.5)$ & 1.5 & $1: 1.5-1.6(1.5)$ \\
\hline VS to $\mathrm{AT} \% *$ & $30-44(38)$ & $40-42$ & $38-43(41)$ \\
\hline Post-vitelline region $\% *$ & $11-14(12)$ & $11-13$ & $12-13(12)$ \\
\hline Post-uterine region $\% *$ & $6.2-10(7.5)$ & $8.1-8.5$ & $6.4-8.4(7.4)$ \\
\hline $\mathrm{SV}$ to AT \%* & $6.7-19(13)$ & $18-19$ & $12-17(15)$ \\
\hline VS to SV \% & $16-25(20)$ & $17-18$ & $19-24(21)$ \\
\hline SV to AT \% of VS to SV & $41-95(63)$ & $102-108$ & $51-88(69)$ \\
\hline
\end{tabular}

ex Kyphosus cornelii (Whitley), Kyphosidae. Stomach. Red Bluff, Kalbarri, Western Australia $\left(27^{\circ} 42^{\prime} \mathrm{S}, 114^{\circ} 10^{\prime} \mathrm{E}\right.$, March 1988). QM G217873, BMNH 1988.6.28.43-52.

ex Kyphosus sydneyanus (Günther), Kyphosidae. Stomach. Off Fremantle, Western Australia $\left(32^{\circ} 07^{\prime} \mathrm{S}, 115^{\circ} 44^{\prime} \mathrm{E}\right.$, March 1988). QM G217874, BMNH 1988.6.28.30-42.

ex Kyphosus sydneyanus ? (Günther), Kyphosidae. Stomach. Ningaloo, Western Australia $\left(22^{\circ} 42^{\prime} \mathrm{S}, 113^{\circ} 40^{\prime} \mathrm{E}\right.$, April 2000). QM G217875-217881, BMNH 2002.4.18.8-12.

ex Kyphosus vaigiensis (Quoy et Gaimard) (Kyphosidae).

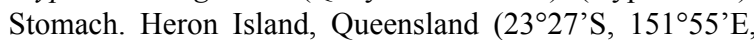
Jan. 1998, April 1999). QM G217882-217886, BMNH 2002.4.18.13-16.

ex Kyphosus vaigiensis (Quoy et Gaimard) (Kyphosidae). Stomach. Moorea, French Polynesia (17³0'S, $149^{\circ} 50^{\prime} \mathrm{W}$, Dec. 1999). QM G217887-217889, BMNH 2002.4.18.1819. ex Kyphosus vaigiensis (Quoy et Gaimard) (Kyphosidae).

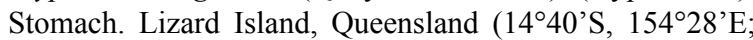
Dec. 2000). QM G217890-217891, BMNH 2002.4.18.17.

Records :

1. Linton (1910); 2. Manter (1947); 3. Sogandares-Bernal (1959); 4. Bravo-Hollis (1965); 5. Manter (1966) 6. Overstreet (1969); 7. Yamaguti (1970); 8. Machida (1980); 9. Hogans et al. (1983); 10. Dyer et al. (1985); 11. Kim et al. (1990); 12. Dyer et al. (1992); 13. León-Règagnon et al. (1996); 14. Lamothe-Argumedo et al. (1997); 15. LeónRègagnon et al. (1997a); 16. León-Règagnon et al. (1997b); 17. Pérez-Ponce de León et al. (1999); 18. Hassanine (2000); 19. Cribb et al. (2001); 20. Present study.

De s c riptions: 1, 2, 4, 7, 8, 18, 20. 
Table 1. Continued.

\begin{tabular}{|c|c|c|}
\hline $\begin{array}{l}\text { Species } \\
\text { Host } \\
\text { Locality } \\
\mathrm{n}\end{array}$ & $\begin{array}{c}\text { Opisthadena dimidia } \\
\text { Kyphosus cinerascens } \\
\text { Heron Island } \\
2\end{array}$ & $\begin{array}{c}\text { Opisthadena dimidia } \\
\text { Kyphosus cinerascens } \\
\text { Ningaloo } \\
9\end{array}$ \\
\hline Length & $6,014-6,150$ & $3,648-6,999(5,267)$ \\
\hline Width & $814-861$ & $474-957(647)$ \\
\hline Forebody & $507-593$ & $377-674(494)$ \\
\hline Pre-oral lobe & $36-42$ & $16-38(27)$ \\
\hline Oral sucker & $224-240 \times 200-206$ & $147-258 \times 131-232(192 \times 171)$ \\
\hline Pharynx & $131-134 \times 142-143$ & $86-150 \times 90-170(109 \times 120)$ \\
\hline Oesophagus & $35-41$ & $9-21(14)$ \\
\hline IB to VS & $119-182$ & $121-233(168)$ \\
\hline Sinus sac & $126-134 \times 126-149$ & $84-150 \times 72-128(110 \times 112)$ \\
\hline GP to VS & $58-129$ & 84-194 (131) \\
\hline Ventral sucker & $629-641 \times 617-634$ & $398-748 \times 399-706(536 \times 515)$ \\
\hline VS to SV & $1,434-1,535$ & $764-1,429(1,068)$ \\
\hline Seminal vesicle & $354-355 \times 243-267$ & $195-387 \times 122-274(282 \times 179)$ \\
\hline $\mathrm{SV}$ to AT & $824-840$ & $573-1,476(1,060)$ \\
\hline Anterior testis & $226-237 \times 295-336$ & $187-239 \times 178-302(213 \times 229)$ \\
\hline Distance between testes & $57-145$ & $23-317(153)$ \\
\hline Posterior testis & $245-253 \times 307-329$ & $194-237 \times 195-278(216 \times 238)$ \\
\hline $\mathrm{PT}$ to $\mathrm{Ov}$ & $406-415$ & $100-351(228)$ \\
\hline Ovary & $252-260 \times 329-355$ & $150-270 \times 184-313(203 \times 257)$ \\
\hline Vitelline masses & $231-264 \times 187-274$ & $157-326 \times 127-365(233 \times 243)$ \\
\hline Post-vitelline region & $708-761$ & $427-745(564)$ \\
\hline Post-uterine region & $382-595$ & $299-442(358)$ \\
\hline Post-caecal region & $102-255$ & $63-219(124)$ \\
\hline Eggs & $41-43 \times 11-14$ & $29-40 \times 10-15(34 \times 13)$ \\
\hline Width \%* & 14 & $11-14(12)$ \\
\hline Forebody \%* & $8.2-10$ & $7.8-11(10)$ \\
\hline Sucker width ratio & $1: 3.0-3.2$ & $1: 2.8-3.1(3.0)$ \\
\hline OS to Pharynx ratio & $1: 1.4-1.5$ & $1: 1.3-1.6(1.4)$ \\
\hline VS to $\mathrm{AT} \% *$ & $43-45$ & $42-49(45)$ \\
\hline Post-vitelline region $\% *$ & 12 & $10-12(11)$ \\
\hline Post-uterine region $\% *$ & $6.3-10$ & $5.5-8.5(6.9)$ \\
\hline $\mathrm{SV}$ to $\mathrm{AT} \% *$ & 14 & $16-23(20)$ \\
\hline VS to SV \% & $23-26$ & $18-22(20)$ \\
\hline SV to AT \% of VS to SV & $54-59$ & $75-112(97)$ \\
\hline
\end{tabular}

* of body length

Abbreviations: AT - anterior testis, GP - genital pore, IB - intestinal bifurcation, Ov - ovary, OS - oral sucker, PT - posterior testis, SV - seminal vesicle, VS - ventral sucker.

D e f i n i t i v e hos t s : Perciformes: Kyphosidae: Kyphosus bigibbus (20), K. cinerascens $(7,8,13,18,19$, 20), $K$. cornelii (20), K. elegans $(3,4,13,17), K$. incisor $(2,13,14,15,16), K$. sectatrix $[?=$ sectarius $](1,2,3,6$, $10,11,12,13), K$. sydneyanus $(5,20), K$. vaigiensis (20), Kyphosus sp. (5); Perciformes: Xiphiidae: Xiphias gladius (9).

D i s t r i b u t i o n : FAO Area 21 NW Atlantic Ocean (9); 31 Off Florida (1, 2, 13), Bimini, British West Indies (3), Biscayne Bay, Florida (6), Puerto Rico (10, 12, 13); 34 Canary Islands (11); 51 Gulf of Aqaba, Red Sea (18); 57 South Australia (5), Western Australia (20); 61 Japan (8, 13); 71 Heron Island, Queensland (5, 19, 20), Lizard Island, Queensland (20); 77 Quintana Roo, Pacific coast
Mexico (13, 14); Chamela Bay, Mexico (15, 16), Jalisco, Mexico (13), Baja California (4, 13, 17), Taboga Island, Pacific coast of Panama (3), Hawaii (7, 13), Moorea, French Polynesia (20).

Remarks. Measurements are given in Table 1. This species has been adequately described by Linton (1910), Manter (1947), Bravo-Hollis (1965), Yamaguti (1970) and Machida (1980), but we have included detailed measurements of our specimens for comparison (Table 1). We can detect no significant differences between our specimens and those described earlier, but we have recoded the matrix in León-Règagnon et al. (1996) in respect of: Character 4, in which we find the testes in either the middle or posterior third of the body-length or 
Table 1. Continued.

\begin{tabular}{|c|c|c|c|}
\hline $\begin{array}{l}\text { Species } \\
\text { Host } \\
\text { Locality } \\
\mathrm{n}\end{array}$ & $\begin{array}{l}\text { Opisthadena dimidia } \\
\text { Kyphosus sydneyanus } \\
\text { off Fremantle } \\
5\end{array}$ & $\begin{array}{c}\text { Opisthadena dimidia } \\
\text { Kyphosus sydneyanus? } \\
\text { Ningaloo } \\
12\end{array}$ & $\begin{array}{c}\text { Opisthadena dimidia } \\
\text { Kyphosus cornelii } \\
\text { Kalbarri } \\
2\end{array}$ \\
\hline Length & $4,264-8,328(6,320)$ & $4,573-7,682(6,129)$ & $2,812-3,706$ \\
\hline Width & $553-1,014(787)$ & $516-898(747)$ & $589-599$ \\
\hline Forebody & $386-545(437)$ & $432-623(563)$ & $387-474$ \\
\hline Pre-oral lobe & $26-42(35)$ & $22-47(35)$ & $37-45$ \\
\hline Oral sucker & $175-267 \times 173-289(216 \times 235)$ & $153-246 \times 140-237(215 \times 199)$ & $175-190 \times 178-207$ \\
\hline Pharynx & $113-207 \times 113-196(165 \times 151)$ & $92-159 \times 94-165(129 \times 138)$ & $117-136 \times 117-121$ \\
\hline Oesophagus & $0-10(2)$ & $7-47(18)$ & $0-13$ \\
\hline IB to VS & $122-215(165)$ & $138-222(179)$ & $113-210$ \\
\hline Sinus sac & $133-226 \times 97-150(177 \times 117)$ & $94-181 \times 88-163(142 \times 138)$ & $133-146 \times 78-82$ \\
\hline GP to VS & $52-97(78)$ & $122-188(149)$ & $65-143$ \\
\hline Ventral sucker & $513-876 \times 468-880(681 \times 670)$ & $438-735 \times 410-703(586 \times 572)$ & $448-487 \times 471-481$ \\
\hline VS to SV & $1,040-1,668(1,289)$ & $786-1,883(1,237)$ & $400-624$ \\
\hline Seminal vesicle & $189-447 \times 112-234(353 \times 191)$ & $141-400 \times 118-270(276 \times 203)$ & $174-254 \times 83-116$ \\
\hline SV to AT & $454-1,197(827)$ & $884-1,653(1,280)$ & $167-333$ \\
\hline Anterior testis & $247-337 \times 186-285(300 \times 238)$ & $171-259 \times 200-279(211 \times 247)$ & $224-261 \times 148-206$ \\
\hline Distance between testes & $0-526(187)$ & $38-385(158)$ & $0-12$ \\
\hline Posterior testis & $272-351 \times 197-328(313 \times 277)$ & $203-282 \times 215-298(233 \times 263)$ & $195-245 \times 219-229$ \\
\hline PT to Ov & $108-479(366)$ & $252-644(375)$ & $0-113$ \\
\hline Ovary & $180-323 \times 179-354(266 \times 294)$ & $179-272 \times 234-366(232-299)$ & $147-210 \times 220-235$ \\
\hline Vitelline masses & $192-353 \times 180-304(297 \times 244)$ & $164-208 \times 301-340(236-263)$ & $203-232 \times 158-186$ \\
\hline Post-vitelline region & $547-1,260(957)$ & $565-902(724)$ & $380-607$ \\
\hline Post-uterine region & $430-734(596)$ & $314-585(459)$ & $363-443$ \\
\hline Post-caecal region & $76-261(140)$ & $95-218(159)$ & $135-231$ \\
\hline Eggs & $36-55 \times 15-19(43 \times 17)$ & $32-40 \times 10-17(36 \times 13)$ & $32 \times 12$ \\
\hline Width \%* & $11-13(13)$ & $11-13(12)$ & 16 \\
\hline Forebody $\% *$ & $6.3-9.1(7.1)$ & $7.7-10(9.3)$ & $10-14$ \\
\hline Sucker width ratio & $1: 2.7-3.0(2.8)$ & $1: 2.71-3.06(2.88)$ & $1: 2.3-2.7$ \\
\hline OS to Pharynx ratio & $1: 1.5-1.7(1.6)$ & $1: 1.38-1.51(1.45)$ & $1: 1.5-1.7$ \\
\hline VS to AT \%* & $36-41(39)$ & $40-49(45)$ & $26-33$ \\
\hline Post-vitelline region $\% *$ & $13-16(15)$ & $10-14(12)$ & $14-16$ \\
\hline Post-uterine region $\% *$ & $8.8-11(10)$ & $6.1-9.2(7.5)$ & $12-13$ \\
\hline SV to AT $\% *$ & $11-14(13)$ & $18-24(21)$ & $5.9-9.0$ \\
\hline VS to SV \% & $18-24(21)$ & $15-25(20)$ & $14-17$ \\
\hline SV to AT \% of VS to SV & $44-76(63)$ & $71-157(107)$ & $42-53$ \\
\hline
\end{tabular}

extending into both regions; $\mathrm{Ch}$. 7, where we find the testes to be tandem; Ch. 8 , where we find that the posterior testis lies more than its own length from the ovary; Ch. 13, where we find papillae around the aperture of the ventral sucker (not used in analysis); and Ch. 20, where we find the vitelline masses to be symmetrical. We follow Overstreet (1969) and Machida (1980) in considering O. cortesi and O. kyphosi, respectively, synonyms of $O$. dimidia. This also means that we do not agree with León-Règagnon et al. (1996) in considering $O$. kyphosi valid, and prefer Machida's (1980) interpretation, feeling that the papillae are so small and easily obscured by some fixation methods that it is inadvisable to rely on this character to distinguish species.
Opisthadena dimidia is now known to have an extremely wide distribution in Kyphosus spp. in the Atlantic, Indian and Pacific Oceans (León-Règagnon et al. 1996). It might well be argued that it is unlikely that this is a single species, as the hosts are not wide-spread pelagic fishes. This could well be the case, but we are unable to detect any morphological features on which a specific separation could be made. This species has been reported in Australian waters before, by Manter (1966), including a mention of it occurring in three species of Kyphosus off Heron Island. All records but one are from Kyphosus spp. and this record from Xiphias gladius in the NW Atlantic (Hogans et al. 1983) is likely to either accidental (Gibson 1996) or erroneous, as only two specimens were recovered from 300 fishes. 
Table 1. Continued.

\begin{tabular}{|c|c|c|c|}
\hline $\begin{array}{l}\text { Species } \\
\text { Host } \\
\text { Locality } \\
n\end{array}$ & $\begin{array}{l}\text { Opisthadena dimidia } \\
\text { Kyphosus bigibbus } \\
\text { Ningaloo } \\
5\end{array}$ & $\begin{array}{c}\text { Mitrostoma nototheniae } \\
\text { Aplodactylus arctidens } \\
\text { Tasmania } \\
2\end{array}$ & $\begin{array}{c}\text { Neopisthadena habei } \\
\text { Kyphosus sydneyanus } \\
\text { off Fremantle } \\
1\end{array}$ \\
\hline Length & $3,959-6,895(5,092)$ & $1,383-2,240$ & 3,535 \\
\hline Width & $479-701(572)$ & $264-404$ & 737 \\
\hline Forebody & $407-574(474)$ & $350-452$ & 825 \\
\hline Pre-oral lobe & $13-31(21)$ & $13-47$ & 44 \\
\hline Oral sucker & $148-209 \times 127-185(173 \times 152)$ & $85-105 \times 76-113$ & $259 \times 318$ \\
\hline Pharynx & $87-125 \times 89-128(99 \times 106)$ & $48-63 \times 53-74$ & $199 \times 194$ \\
\hline Oesophagus & $8-22(16)$ & $0-24$ & 0 \\
\hline IB to VS & $147-255(186)$ & $231-245$ & 507 \\
\hline Sinus sac & $93-148 \times 78-136(115 \times 104)$ & $72-100 \times 39-47$ & $297 \times 137$ \\
\hline GP to VS & $98-180(130)$ & $149-204$ & 403 \\
\hline Ventral sucker & $392-588 \times 387-581(470 \times 461)$ & $132-207 \times 162-254$ & $560 \times 502$ \\
\hline VS to SV & $928-1,491(1,149)$ & $76-152$ & 148 \\
\hline Seminal vesicle & $126-331 \times 86-239(204 \times 138)$ & $80-161 \times 39-121$ & $1,255 \times 75$ \\
\hline SV to AT & $746-1,510(1,061)$ & $69-117$ & 0 \\
\hline Anterior testis & $204-253 \times 169-255(218 \times 204)$ & $103-112 \times 84-145$ & $282 \times 309$ \\
\hline Distance between testes & $21-313(112)$ & $38-117$ & 0 \\
\hline Posterior testis & $205-265 \times 181-267(224 \times 216)$ & $106-133 \times 97-133$ & $307 \times 299$ \\
\hline $\mathrm{PT}$ to $\mathrm{Ov}$ & $155-450(259)$ & $44-141$ & 45 \\
\hline Ovary & $151-245 \times 174-307(200 \times 235)$ & $106-134 \times 110-134$ & $145 \times 245$ \\
\hline Vitelline masses & $143-299 \times 106-273(221 \times 183)$ & $81-134 \times 75-140$ & $194-204 \times 225-284$ \\
\hline Post-vitelline region & $382-704(515)$ & $172-320$ & 652 \\
\hline Post-uterine region & $259-429(329)$ & $131-167$ & 375 \\
\hline Post-caecal region & $56-238(117)$ & $66-85$ & $89-153$ \\
\hline Eggs & $27-40 \times 9-15(31 \times 11)$ & $22-31 \times 9-14(26 \times 12)$ & $28 \times 14$ \\
\hline Width \%* & $10-13(11)$ & $18-19$ & 21 \\
\hline Forebody $\% *$ & $7.9-10(10)$ & $20-25$ & 23 \\
\hline Sucker width ratio & $1: 2.9-3.1(3.0)$ & $1: 2.1-2.3$ & $1: 1.6$ \\
\hline OS to Pharynx ratio & $1: 1.4-1.5(1.4)$ & $1: 1.4-1.5$ & $1: 1.6$ \\
\hline VS to AT \%* & $45-50(47)$ & $16-19$ & 12 \\
\hline Post-vitelline region $\% *$ & $9.2-11(10)$ & $12-14$ & 18 \\
\hline Post-uterine region $\% *$ & $5.6-7.9(6.6)$ & $7.5-9.4$ & 11 \\
\hline SV to AT \%* & $17-24(20)$ & $5.0-5.2$ & 0 \\
\hline VS to SV \% & $22-24(23)$ & $5.5-6.8$ & 4.2 \\
\hline $\mathrm{SV}$ to AT $\%$ of VS to SV & $74-110(90)$ & $77-90$ & 0 \\
\hline
\end{tabular}

* of body length

Abbreviations: AT - anterior testis, GP - genital pore, IB - intestinal bifurcation, Ov - ovary, OS - oral sucker, PT - posterior testis, SV - seminal vesicle, VS - ventral sucker.

\section{Neopisthadena habei Machida, 1980}

Fig. 3

T y p e-host and locality: Kyphosus cinerascens, Kii Peninsula, Japan.

$\mathrm{New}$ record:

Ex Kyphosus sydneyanus (Günther), Kyphosidae. Stomach. Off Fremantle, Western Australia (32 $07^{\prime} \mathrm{S}, 115^{\circ} 44^{\prime} \mathrm{E}$, March 1988). BMNH 1988.6.28.29.

Records :

1. Machida (1980); 2, Hafeezullah (1990); 3. LeónRègagnon et al. (1996); 4. Present study.

Des criptions: $1,2,4$.

D e fin it ive hos ts: Kyphosidae: Kyphosus cinerascens $(1,2,3)$, K. sydneyanus (4).
D i s t r i b u t i o n : FAO Area 51 Gulf of Mannar (2); 57 off Western Australia (4); 61 off Japan $(1,3)$.

Remarks. Measurements are given in Table 1. This is the fourth record of this species (see above). Machida (1980) and Hafeezullah (1990) presented good descriptions from which the single specimen we have available does not differ significantly, apart from being considerably smaller. This may be a juvenile form as the relatively few eggs appear to be poorly formed and there are many small globules of egg-shell-like material in the uterus.

Hassanine (2000) described Opisthadena dimidia from Kyphosus cinerascens from the Red Sea and stated 
that the variation found encompassed the features of Neopisthadena. We doubt this finding, as none of the 54 specimens we have seen, from a wide geographical range, shows this type of variation and we consider it possible that Hassanine (2000) had a mixed sample.

Checking the matrix in León-Règagnon et al. (1996) against our observations and the description of Hafeezullah (1990) has suggested the following changes: Character 7, we found the testes tandem; Ch. 8 , the distance between the posterior testis and the ovary varies between both character states.

\section{PHYLOGENETIC RELATIONSHIPS}

We have based our cladistic study on the matrix presented by León-Règagnon et al. (1996), but have included four new characters. We have also recoded some characters of the species (see below). The matrix is shown as Table 2. The parsimony searches in PAUP* were exhaustive, with Genolinea and Neotheletrum as outgroups and all characters were unordered. A single most parsimonious tree of length 26 was recovered (Fig. 4).

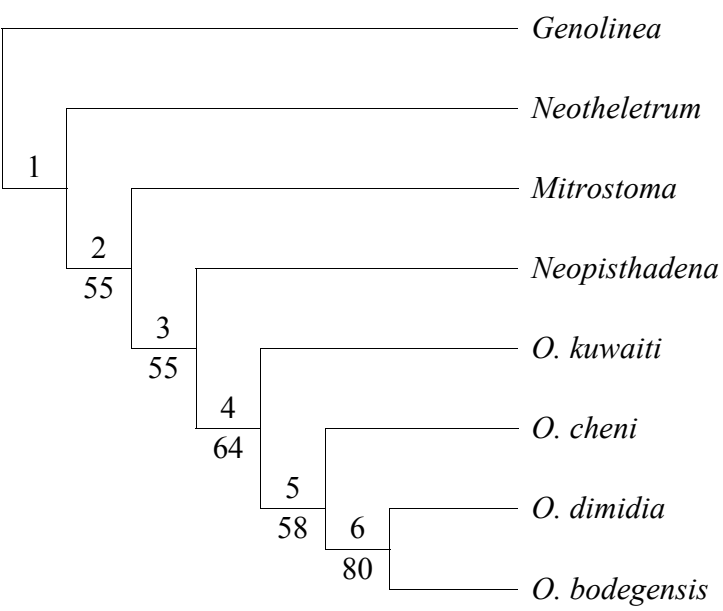

Fig. 4. Phylogeny of Opisthadena and related forms. The single most parsimonious tree based on 21 characters, with Genolinea and Neotheletrum as outgroups. Characters unordered. Tree length 26. CI $=0.769$, CI excluding uninformative characters $=0.549, \mathrm{RI}=0.714, \mathrm{HI}=0.231$. Internode numbers are given above the internodes and Bootstrap support figures (1000 replicates) below. Character support for each internode and terminal taxon is defined below (numbered characters) and includes apomorphy (A), homoplasy $(\mathrm{H})$ or reversal (R): 1: (none); $2:\left(\mathrm{A}: 7^{2}, 9^{1}\right)$; 3: (A: 16 $)$; 4: (A: ? $\left.1^{2}, 12^{1}, 21^{0}, 23^{0}\right)$; 5: (A: $\left.3^{1}\right)$; 6: (A: $\left.5^{1}, 14^{1}\right)$; Neotheletrum: (A: $\left.7^{0}, 15^{0} ; \mathrm{H}: 9^{0}, 24^{1}\right)$; Mitrostoma: (A: $18^{1} ; \mathrm{H}$ : $\left.10^{1}\right)$; Neopisthadena: (A: $\left.12^{0} ; \mathrm{H}: 9^{0}, 22^{1}\right)$; Opisthadena kuwaiti: (A: $\left.2^{1}, 11^{1} ; \mathrm{H}: 9^{0}, 22^{1}, 24^{1}\right)$; O. cheni: $\left(\mathrm{H}: 10^{1}, 22^{1}\right)$; O. dimidia: (A: ?4 $\left.4^{1} ; \mathrm{R}: 3^{0}\right) ; O$. bodegensis: (none).

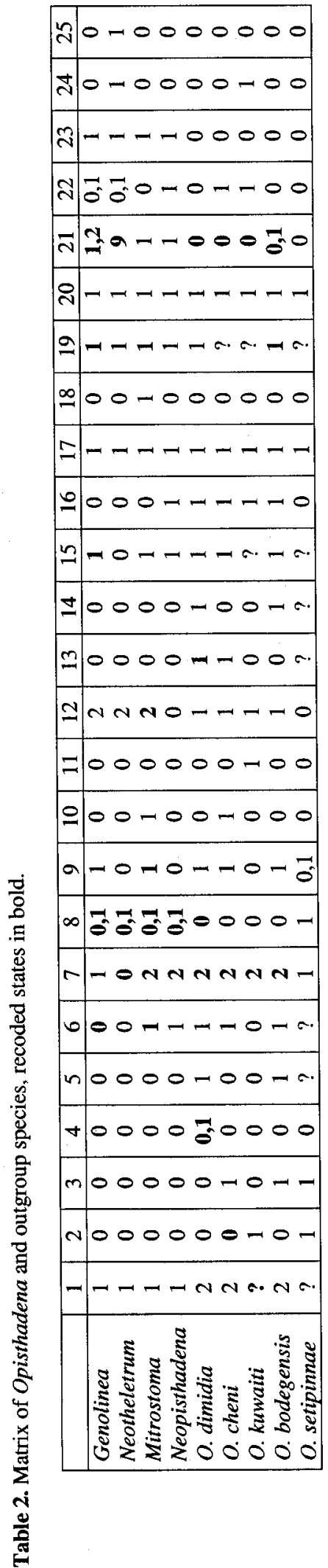




\section{Character argumentation}

Characters 1-21 are from León-Règagnon et al. (1996), 22-25 are new. Four characters listed by LeónRègagnon et al. (1996) are not used in the analysis, but are retained in this list and matrix to allow the numbering of the characters to be retained. These characters are numbers 6 and 13, where we believe that the observation of these papillae is unreliable (see above) and characters 17 and 20, which are invariant in the species analysed.

(1) Seminal vesicle: 0 , saccate; 1 , tubular; 2 , seminal sac. (2) Egg size: 0, $>25 \mu \mathrm{m} ; 1,13-18 \mu \mathrm{m}$. (3) Pharynx size: 0 , pharynx smaller than the oral sucker; 1 , pharynx and oral sucker of similar size. (4) Testes location: 0 , in middle third of body; 1 , in posterior third of body. (5) Excretory ducts: 0, not branched anteriorly to the ventral sucker; 1, branched anteriorly. (6) Oral papillae: 0 , absent; 1 , present. (7) Testes position: 0, symmetrical; 1, oblique; 2, tandem. (8) Distance between posterior testis and ovary: 0, posterior testis lies more than its own length from the ovary; 1, posterior testis lies less than its own length from the ovary. (9) Location of vitellarium and ovary: 0 , in third quarter of body; 1 , in fourth quarter of body. (10) Muscular sphincter around ventral sucker: 0, absent; 1, present. (11) Seminal vesicle wall: 0 , thin; 1 , thick. (12) Anterior extremity of pars prostatica: 0 , at level of ventral sucker; 1 , in hindbody; 2, in forebody. (13) Papillae around aperture of ventral sucker: 0, absent; 1, present. (14) Extension of excretory ducts: 0 , running anteriorly of ventral sucker; 1 , anterior to ventral sucker, but with branches running posteriorly to testes level. (15) Transverse fold in anterior hindbody: 0, absent; 1, present. (16) Distinct ejaculatory duct: 0 , absent; 1, present. (17) Blind seminal receptacle: 0 , absent; 1, present. (18) Presomatic pit: 0, absent; 1, present. (19) Excretory vesicle bifurcation: 0 , near posterior margin of ventral sucker; 1, at level of testes. (20) Uterine seminal receptacle: 0, present; 1, absent. (21) Vitelline masses configuration: 0 , symmetrical; 1 , oblique; 2 , tandem. (22) Sucker-width ratio: $0,>1: 2 ; 1,<1: 2$. (23) Forebody as proportion of body length: $0,<18 \% ; 1,>19 \%$. (24) Uterus: 0 , pre-dominantly pre-ovarian, 1 , about equally pre- and post-ovarian, 2, predominantly post-ovarian. (25) Vitelline masses: 0, 2; 1, 3.

\section{Observations on other species}

Opisthadena cheni Martin, 1978. According to our interpretation of Martin (1978) we have recoded the following characters: Character 2, the eggs are quoted as being 28-44 long; Ch. 7, the testes appear more or less tandem in the figure; Ch. 21, the vitelline masses appear to be symmetrical and are described as transverse.

Opisthadena kuwaiti Al-Yamani et Nahhas, 1981. We have recoded a few characters on the basis of our interpretation of Al-Yamani and Nahhas (1981): Character 1 , we query the status of the seminal vesicle, although it seems likely that the original coding is correct; Ch. 7 the testes are described and figured as tandem; Ch. 21, the vitelline masses are described as 'side by side' and illustrated as symmetrical (in the specialised meaning associated with digenean description).

Opisthadena bodegensis Johnson et Copsey, 1953. We have recoded a few characters based on our reading of Johnson and Copsey (1953) and observations of the type-specimen by Patricia Pilitt and Eric Hoberg (pers. comm.): Character 7, the testes are described as slightly tandem, but appear oblique in the specimen and have been coded as such; Ch. 19, the excretory vesicle is described and figured as bifurcating between the testes; Ch. 21, the vitelline masses are described as 'side by side' and are symmetrical in the type-specimen.

Opisthadena setipinnae Qiu et Liang in Shen and Qiu, 1995. This species is reported in the engraulid Setipinna taty from Tianjin Tanggu, China (Shen and Qiu 1995). It is coded for the matrix, but as several character states are not available in the description, it was not included in the final analysis. On the other hand, the species $O$. fujianensis Tang, Shi, Cao, Guan et Pan, 1983 and O. marina Tang, Shi, Cao, Guan et Pan, 1983 are not included because, following LeónRègagnon et al. (1996), we consider these forms species inquirendae.

Our observations on Neotheletrum Gibson et Bray, 1979 come from the descriptions of the type-species $N$. lissosomum (Manter, 1940) and N. gravidum (Manter, 1940), N. magnasaccum (Sogandares-Bernal et Sogandares, 1961) and N. pomacentri (Nahhas et Cable, 1964) (Bray and Cribb 2000, Manter 1940, Nahhas and Cable 1964, Sogandares-Bernal and Sogandares 1961), plus the observations of León-Règagnon et al. (1996). We have recoded: Character 7 , as all descriptions indicate that the testes are 'oblique' or 'diagonal'; Ch. 8, where it appears that both character states apply; and Ch. 21, which is considered inapplicable to this genus, as it has three vitelline masses.

Our observations on Genolinea Manter, 1925 are based on examination of numerous specimens of the type-species, G. laticauda Manter, 1925, from the northern Atlantic Ocean ${ }^{1}$ (northern Pacific forms may belong to a distinct species, G. anura (Layman, 1930) according to Gibson 1996). We have recoded the following: Character 6, we have been unable to detect oral papillae, the oral sucker is invested with a hood-like fold, something like that found in Mitrostoma (this character was not used in the analysis); Ch. 8, the posterior testis in fully-gravid specimens of $G$. laticauda may be more than its own length from the ovary, although in most specimens they are adjacent or contiguous; Ch. 15, a transverse tegumental fold is present closely posterior to the ventral sucker; Ch. 19, in several whole-mounts the bifurcation of the excretory vesicle can be seen at the level of the anterior part of the

\footnotetext{
${ }^{1}$ See p. 288
} 
anterior testis or just anterior to it, and in one set of serial sections it appears to be about halfway between the anterior testis and the ventral sucker, rather than close to the ventral sucker; and Ch. 21, in these specimens the vitelline masses range from virtually symmetrical to tandem, many are diagonally oblique.

\section{Relationships}

The single most parsimonious tree derived from our analysis is shown as Fig. 4. In the legend we summarise the apomorphies, homoplasies and reversal which can be deduced from this tree. This analysis has highlighted problems with several of the characters. The distinct sphincter around the ventral sucker aperture is a case in point. It is most distinctive in Mitrostoma and is coded as present in $O$. cheni due to the original description. All of these species do, however, have concentric muscles around the aperture, but in most cases these are not well enough developed for them to be considered a distinct sphincter, but it is very much a case of individual interpretation. Similarly the presence of an ejaculatory duct is a matter of some interpretation. Even if the pars prostatica reaches close to the sinus-sac it may have a short, gland-less duct between, which might be interpreted as an ejaculatory duct.

Our tree (Fig. 4) is mostly congruent with the single most parsimonious tree of León-Règagnon et al. (1996), with Neopisthadena, the sister-group to the monophyletic Opisthadena. O. setipinnae, not included by León-Règagnon et al. (1996) and not included in our final analysis, was resolved in a preliminary analysis (not shown) as the sister taxon to $O$. bodegensis but is dissimilar in its host, an engraulid clupeiform rather than a stichaeid perciform, and its distribution off China rather than California. León-Règagnon et al. (1996) pointed out that $O$. dimidia was very widespread, but that unlike other widespread digeneans such as Derogenes varicus (Müller, 1780), it was restricted to one genus of hosts. Substantially, this remains the case and our evidence merely emphasises the widespread nature of the species, suggesting perhaps that the distribution is likely to be more or less continuous, and that of the scenarios suggested by León-Règagnon et al. (1996) the idea that its occurrence in the Caribbean Sea is anthropogenic is unlikely to be true. We believe that the records of Mitrostoma in the omnivore Paranotothenia magellanica are likely to be erroneous, and that the major (perhaps sole) host is the largely herbivorous fish Aplodactylus arctidens. This indicates that the species under study here are mostly parasites of herbivorous fishes, although these fishes are not all closely related. The hosts of Neopisthadena and $O$. dimidia (syn. $O$. kyphosi) are herbivorous Kyphosus spp., and that of $O$. cheni, the related herbivore Girella nigricans. The host of $O$. kuwaiti is the herbivorous and detritivorous mugilid Valamugil seheli. O. setipinnae is anomalous, in that its engraulid host, Setipinna taty, is a schooling omnivore. As León-Règagnon et al. (1996) pointed out, the data we have at present leave many questions as to the evolutionary associations in this group of parasites unresolved.

Acknowledgements. We are grateful to Ingo Ernst, Cedric Lo, Sylvie Pichelin and Alan Williams for assistance with the collection and Trudy Wright for the preparation of the specimens. The work was supported by ARC, ABRS and the Heron Island Research Station. Thanks are also due to Patricia Pilitt and Eric Hoberg for examining the holotype of Opisthadena bodegensis in the USNPC, Pete Olson who performed the PAUP* analyses and David Gibson who read the manuscript.

\footnotetext{
${ }^{1}$ The details of the Genolinea laticauda specimens examined are as follows: ex Glyptocephalus cynoglossus (L.), Pleuronectidae, stomach, Hamilton Inlet Bank, Canada $\left(54^{\circ} 07^{\prime} \mathrm{N}, 54^{\circ} 26^{\prime} \mathrm{W}\right.$, depth $192 \mathrm{~m}$, July 1975) (2 specimens); ex Hemitripterus americanus Cuvier, Hemitripteridae, stomach, Passamaquoddy Bay, New

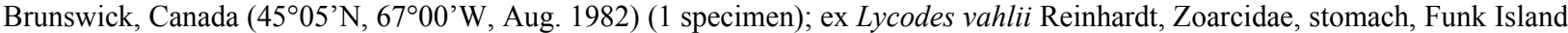
Bank, Canada (51 ${ }^{\circ} 12^{\prime} \mathrm{N}, 51^{\circ} 38^{\prime} \mathrm{W}$, July 1975) (1 specimen); ex Macrourus berglax Lacepède, Macrouridae, stomach, off Rona,

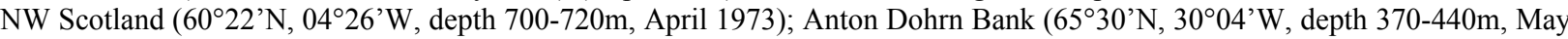

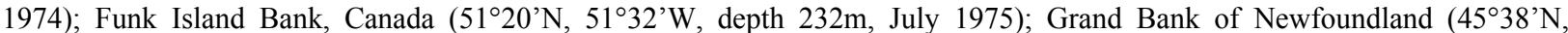
$48^{\circ} 16^{\prime} \mathrm{W}$, depth 715-720m, May 1981). (21 specimens, including 1 set of serial sections); ex Microstomus kitt (Walbaum), Pleuronectidae, stomach, off Faeroes $\left(62^{\circ} \mathrm{N}, 07^{\prime} \mathrm{W}\right)$. (4 specimens); ex Myoxocephalus octodecemspinosus (Mitchill), Cottidae, stomach, Passamaquoddy Bay, New Brunswick, Canada, $\left(45^{\circ} 05^{\prime} \mathrm{N}, 67^{\circ} 00^{\prime} \mathrm{W}\right.$, Aug. 1982) (7 specimens); ex Pseudopleuronectes americanus (Walbaum), Pleuronectidae, stomach, Passamaquoddy Bay, New Brunswick, Canada, $\left(45^{\circ} 05^{\prime} \mathrm{N}, 67^{\circ} 00^{\prime} \mathrm{W}\right.$, Aug. 1982) (3 specimens); ex Trachyrincus scabrus (Rafinesque), Macrouridae, stomach, Bay of Biscay $\left(47^{\circ} 02^{\prime} \mathrm{N}, 05^{\circ} 37^{\prime} \mathrm{W}\right.$, depth 330-610m, Jan. 1971) (2 specimens); ex Urophycis chuss (Walbaum), Phycidae, stomach, Passamaquoddy Bay, New Brunswick, Canada $\left(45^{\circ} 05^{\prime} \mathrm{N}, 67^{\circ} 00^{\prime} \mathrm{W}\right.$, Aug. 1982) (4 specimens); ex Urophycis tenuis (Mitchill), Phycidae, Scotian Shelf, Canada (1 specimen)
} 


\section{REFERENCES}

AL-YAMANI F.Y., NAHHAS F.M. 1981: Digenetic trematodes of marine fishes from the Kuwaiti Coast of the Arabian Gulf. Kuwait Bull. Mar. Sci. 3: 1-22.

BRAVO-HOLLIS M. 1965: Helmintos de peces de aguas mexicanas del Pacífico. XXIV. Descripción de Opisthadena cortesi n. sp. (Tremátodo). An. Inst. Biol., Univ. Nac. Autón. Mexico 36: 141-145.

BRAY R.A., CRIBB T.H. 2000: The status of the genera Hysterolecithoides Yamaguti, 1934, Neotheletrum Gibson \& Bray, 1979 and Machidatrema León-Règagnon, 1998 (Digenea: Hemiuroidea), including a description of $M$. leonae n. sp. from Australian waters. Syst. Parasitol. 46: 122.

BROOKS D.R., O'GRADY R.T., GLEN D.R. 1985: Phylogenetic analysis of the Digenea (Platyhelminthes: Cercomeria) with comments on their adaptive radiation. Can. J. Zool. 63: 411-443.

CRIBB T.H., BRAY R.A., LITTLEWOOD D.T.J., PICHELIN S., HERNIOU E.A. 2001: The Digenea. In: D.T.J. Littlewood and R.A. Bray (Eds.), Interrelationships of the Platyhelminthes. Taylor \& Francis, London, pp. 168-185.

DYER W.G., WILLIAMS E.H., BUNKLEY-WILLIAMS L. 1992: Homalometron dowgialloi sp. n. (Homalometridae) from Haemulon flavolineatum and additional records of digenetic trematodes of marine fishes in the West Indies. J. Helminthol. Soc. Wash. 59: 182-189.

DYER W.G., WILLIAMS E.H., WILLIAMS L.B. 1985: Digenetic trematodes of marine fishes of the western and southwestern coasts of Puerto Rico. Proc. Helminthol. Soc. Wash. 52: 85-94.

FROESE R., PAULY D. 2001: FishBase. World Wide Web electronic publication. www.fishbase.org.

GIBSON D.I. 1996: Trematoda. In: L. Margolis and Z. Kabata (Eds.), Guide to the parasites of fishes of Canada, Part IV. NRC Research Press, Canadian Special Publication of Fisheries and Aquatic Sciences, 124, Ottawa, pp. 1-373.

GIBSON D.I. 2002: Family Hemiuridae Looss, 1899. In: D.I. Gibson, A. Jones and R.A. Bray (Eds.), Keys to the Trematoda. Volume 1. CAB International, Wallingford, pp. 307-340.

GIBSON D.I., BRAY R.A. 1979: The Hemiuroidea: terminology, systematics and evolution. Bull. Br. Mus. (Nat. Hist.) (Zool. Ser.). 36: 35-146.

HAFEEZULLAH M. 1990: Digenetic trematodes of marine fishes of India (superfamily Hemiuroidea: families Lecithasteridae and Bunocotylidae). Rec. Zool. Surv. India 86: 493-504.

HASSANINE R.M.E.-S. 2000: On two digenetic trematodes (Family: Hemiuridae Looss, 1899) from some fish in the Gulf of Aqaba, with a discussion on the validity of their genera. J. Egypt. German Soc. Zool. 33(D): Invertebrate Zoology and Parasitology, 45-55.

HOGANS W.E., BRATTEY J., UHAZY L.S., HURLEY P.C. 1983: Helminth parasites of swordfish (Xiphias gladius L.) from the northwest Atlantic Ocean. J. Parasitol. 69: 11781179 .
JOHNSON W.F., COPSEY J.E. 1953: Opisthadena bodegensis n. sp. (Trematoda) from Dillon Beach, California. Trans. Am. Microsc. Soc. 72: 78-81.

KIM M., GIJÓN-BOTELLA H., LÓPEZ-ROMÁN R. 1990: The Bunocotylidae, Derogenidae, Hemiuridae and Azygiidae of marine fishes. Bull. Soc. Fr. Parasitol. 8: (VII International Congress of Parasitology, Paris) Supplement 2, p. 733.

LAMOTHE-ARGUMEDO R., GARCÍA-PRIETO L., OSORIO-SARABIA D., PÉREZ-PONCE de LEÓN G. 1997: Catálogo de la Colección Nacional de Helmintos. Universidad Nacional Autónoma de México, México, 211 pp.

LEÓN-RÈGAGNON V., PÉREZ-PONCE de LEÓN G., BROOKS D.R. 1996: Phylogenetic analysis of Opisthadena (Digenea: Hemiuridae). J. Parasitol. 82: 1005-1010.

LEÓN-RĖGAGNON V., PÉREZ-PONCE de LEÓN G., BROOKS D.R. 1998: Phylogenetic analysis of the Bunocotylinae Dollfus, 1950 (Digenea: Hemiuridae). J. Parasitol. 84: 147-152.

LEÓN-RÈGAGNON V., PÉREZ-PONCE de LEÓN G., GARCÍA-PRIETO L. 1997a: Description of Heteroplectanum oliveri sp.n. (Monogenea: Diplectanidae) and comments on the helminth fauna of Kyphosus elegans (Perciformes: Kyphosidae) from Chamela Bay, México. J. Helminthol. Soc. Wash. 64: 9-16.

LEÓN-RÈGAGNON V., PÉREZ-PONCE de LEÓN G., LAMOTHE-ARGUMEDO R. 1997b: Hemiuriformes de peces marinos de la Bahía de Chamela, México, con la descripción de una nueva especie del género Hysterolecitha (Digenea: Hemiuridae: Lecithasterinae). An. Inst. Biol. Univ. Nac. Autón. México, Series Zoología, 68: 134.

LINTON E. 1910: Helminth fauna of the dry Tortugas II. Trematodes. Pap. Tortugas Lab. Carnegie Inst. Wash. 4: 11-98.

MACHIDA M. 1980: Hemiurid trematodes of Kyphosus collected around Cape Shionomisaki, Kii Peninsula. Mem. Natl. Sci. Mus., Tokyo 13: 113-120.

MANTER H.W. 1940: Digenetic trematodes of fishes from the Galapagos Islands and the neighboring Pacific. Allan Hancock Pacif. Exped. 2: 325-497.

MANTER H.W. 1947: The digenetic trematodes of marine fishes of Tortugas, Florida. Am. Midl. Nat. 38: 257-416.

MANTER H.W. 1954: Some digenetic trematodes from fishes of New Zealand. Trans. R. Soc. N.Z. 82: 475-568.

MANTER H.W. 1966: A peculiar trematode, Gorgocephalus kyphosi gen. et sp. n. (Lepocreadiidae: Gorgocephalinae subfam. n.), from a marine fish of South Australia. J. Parasitol. 52: 347-350.

MANTER H.W., CROWCROFT P.W. 1950: A new genus of amphistome (Trematoda) from a Tasmanian marine fish. Proc. Helminthol. Soc. Wash. 17: 122-126.

MARTIN C. 1978: Digenetic trematodes of the marine fish, Girella nigricans (Ayres), from southern California with a description of two new species. Proc. Helminthol. Soc. Wash. 45: 175-181. 
NAHHAS F.M., CABLE R.M. 1964: Digenetic and aspidogastrid trematodes from marine fishes of Curaçao and Jamaica. Tulane Stud. Zool. 11: 169-228.

OVERSTREET R.M. 1969: Digenetic trematodes of marine teleost fishes from Biscayne Bay, Florida. Tulane Stud. Zool. Bot. 15: 119-176.

PÉREZ-PONCE de LEÓN G., GARCÍA-PRIETO L., MENDOZA-GARFIAS B., LEÓN-RĖGAGNON V., PULIDO-FLORES G., ARANDA-CRUZ C., GARCÍAVARGAS F. 1999: Listados faunísticos de México. IX. Biodiversidad de helmintos parásitos de peces marinos y estuarinos de la Bahía de Chamela, Jalisco. Universidad Nacional Autónoma de México, México, 51 pp.

Received 23 January 2002
SHEN J.-W., QIU Z.-Z. 1995: Studies on the trematodes of fishes from the Yellow Sea and the Bo Hai Sea. Science Press, Beijing, 207 pp. (In Chinese.)

SOGANDARES-BERNAL F. 1959: Digenetic trematodes of marine fishes from the Gulf of Panama and Bimini, British West Indies. Tulane Stud. Zool. 7: 69-117.

SOGANDARES-BERNAL F., SOGANDARES L.M. 1961: Nine digenetic trematodes of marine fishes from the Atlantic coast of Panama. Tulane Stud. Zool. 8: 141-153.

SWOFFORD D.L. 1998: PAUP*. Phylogenetic Analysis Using Parsimony *and Other Methods. Sinauer Associates, Sunderland, Massachusetts, USA.

YAMAGUTI S. 1970: Digenetic Trematodes of Hawaiian Fishes. Keigaku Publishing Co., Tokyo, 436 pp.

Accepted 14 May 2002 
Bray, Cribb: Opisthadena and related genera 